\title{
A Social Networking-based Advertising to Enhance Customer Reach Target
}

\author{
Ahmad Najmi Amer Haider Nuar", Hairulnizam Mahdin", Noryusliza Abdullah", \\ Rozanawati Darman", Masitah Ahmad ${ }^{*}$ \\ ${ }^{\#}$ Faculty of Computer Science and Information Technology Universiti Tun Hussein Onn Malaysia, \\ Parit Raja, 86400 Batu Pahat, Johor, Malaysia \\ E-mail:hairuln@uthm.edu.my \\ *Faculty of Information Management, Universiti Teknologi Mara, \\ 40150, Selangor, Malaysia.
}

\begin{abstract}
A traditional advertising is a method to deliver commercial messages to mass audiences through the newspaper, outdoor billboards, radio, and television. This method is quite expensive for the small and medium company. The new concept of advertising such as social media, website, and application provide an inexpensive way to promote businesses. The proposed idea is to create a new platform for advertising and promotional tools which is called Tagme. This system is developed based on web environment on Windows and Android. Tagme allows marketers to promote their event, business or store and give promotions including vouchers to customers. Tagme also will notify customers instantly with any events or promotions such as free voucher giveaway that will be managed by the marketers themselves. Tagme provides a form of solution for marketers to promote their business efficiently as it provides web analytics and users preferences functions. This will allow marketers to promote their business to specific and focused customers.
\end{abstract}

Keywords — social network; android apps; online marketing

\section{INTRODUCTION}

The emergence of apps on smartphones has changed the way we do things. The apps act as one stops center that gathers all resources in its scope to be available at user finger points. Takes Uber apps, for example, the mechanism of finding available transportation has never been easier than before. It allows users to save a lot of money and time instead of walking around to hail a taxi. Apps cover not only business, but also security [1], foods [2], and social aspects of our life [3]. The social networking apps, such as Facebook can change the way we connect with our friends. It's even been used as online learning tools [4], and it is also very popular medium to promote products to the intended users based on their profile [5]. Based on this scenario we proposed a new model of business promotions through the use of apps and social networking. Traditional advertisements such as banner, coupon or brochure are not effective as they may not reach to the intended target group. This is because the retailer does not know if the traditional advertisement reaches to their intended customers since there is no technique or specific system that can calculate the effectiveness of the traditional advertisement. Furthermore, there is the possibility of misplacing or forgetting to use a coupon which frequently happens among the customers since the state of the coupon itself is small and made of paper that can be crumpled [6]. This will lead to paper waste and make the retailer loss a certain amount of money that has been spent on marketing. Besides, by using traditional advertisement, customer's navigation behaviour or preferences while doing online shopping cannot be easily detected and controlled since consumer behaviour is difficult to predict. This behaviour is important data for analytics used as to understand the consumer purchase behaviour. By studying consumer purchase behaviour, decision-making in the process of purchase can be done in order to discover purchasing motives and purchasing frequency [7]. The use of social networking can provide us with such data where friends will only probably tag their friends that have interest in the same products. By tagging their friends, business owners also can offer some discount to both tagged and tagger to lure them into buying their product.

This application developed for marketers and store owners around Batu Pahat, Johor Malaysia. This application has two target users which are: Store owners and the customer. The store owners who are also known as the 
retailer will be able to update their events and sales information to keep their customers up-to-date with their latest promotions and to direct customers coming to their shop. All of this can be done inside the web dashboard. The customers can view the latest promotions and advertisements on their phone and tag their friend into shopping activities. Plus, this application can be integrated with Facebook as to connect the user with their friends. This application requires an internet connection to access the content of the apps.

This app is expected to reduce the paper advertisements by notifying the customers with the latest update of promotions and events through the apps. This will give a big advantage to the retailer for marketing as they can save money for printing out vouchers and brochures. This also will allow customers to plan ahead their schedule to shop together with their friends. This will help retailers to promote their store through social networking based application. Therefore, this concept will encourage more customers to come to their store as they need to redeem the voucher inside the store. They just need to bring their smartphone and show the electronic coupon to store owner. The store owner will take the unique number inside the electronic coupon and validate it via system dashboard.

The importance of this project is this application can improve sales of the retailers since it has data analysis functionality of customer behaviors. By analysing this behaviour, the retailer can understand customer purchase behaviour and leverage it to maximise profit and reach to intended customer. This application can provide the customer the best offer or information from retailers. This application can help the retailer to save a lot of money since the cost of advertisement is cheaper and affordable. Plus,

The paper is organized as follows: Section II will discuss on Material and Method, Section III is on implementation, Section III presents the results and discussion and Section IV is the conclusion.

\section{MATERIAL AND METHOD}

During the development of the mobile application and a web-based system for Tagme, the main components are the developing an application that will integrate shopping experience and social network into an online shopping community. Besides, comparison of existing similar system is made to analyses the pros and cons of this system.

Social networks have made a significant impact on how today's Internet users communicate, search for and share data. Facebook is one of the social network's platforms that plays a big role in changing the way of users communicate with each other. Plus, with the new improvement that has been made on Facebook, they manage to become a new marketing communication tools that provide opportunities for companies to influence their brands toward consumers [8].

Numerous proposals have been made to improve existing distributed systems by leveraging the inherent trust built into social links. Many believe that augmenting online marketplaces with social networking should improve trust between transaction partners and improve user satisfaction [9].

\section{A. Technology for Developing Web and Mobile Application}

The development of Tagme will require a different type of front-end framework. For the website and mobile development, the front-end framework that will be used is Bootstrap. Bootstrap is a sleek, intuitive and powerful mobile first front-end for faster and easier web development [10]. It contains HTML and CSS-based design templates for typography, forms, buttons, navigation, and other interface components. Bootstrap provides a good user interface since Bootstrap has their own libraries for certain components such as a button, panel, forms, and input group. The apps will be developed using Eclipse ADT, which is a software that purposely for developing an Android application. Eclipse ADT provides a workspace for application development and has extensive plugins that allow users to upgrade the environment. Eclipse ADT was also written in Java language. For the development of mobile application using Eclipse ADT, Tagme is categorized as a hybrid application. Hybrid applications are the result of two join platform which are native apps and web apps. Web apps are referring to the web page that looks like an app if the web page is run inside a smartphone using a web browser. Native apps are referring to an app that is developed for specific mobile's operating system such as Android and iOS. Hybrid apps are chosen because they allow cross-platform development and thus significantly reduce development costs: that is, the same HTML code components can be reused on different mobile operating systems. For the development of web system, Angular.js will be used. The framework adapts and extends traditional HTML to serve dynamic content through two-way data-binding that allows for the automatic synchronization of models and views. To make the data is integrated with the database, the use of PHP is required. PHP is a server-side scripting language designed for web development but also used as a general-purpose programming language. PHP code can be simply mixed with HTML code, or it can be used in combination with various template engines and web frameworks. The technology of database that will be used is MySQL since it is easy to be configured and user-friendly.

\section{B. Reviews on Similar System}

All of the technology that has been used by Groupon and LivingSocial was released by BuiltWith. BuiltWith is a website profiler tool that provides reports regarding analytics, technologies, hosting and traffic.

Groupon is a website that provides a deal-of-the-day that features discounted gift certificates for the customer. Groupon was launched in November 2008, and the first market for Groupon was Chicago [11]. To use the coupon's promotion that provided by Groupon, the customer must pay the initial fees. This coupon is valid up to a year and can be redeemed during that duration. Plus, each promotion that promoted by Groupon only valid after a certain minimum number of customer purchase the deals. Groupon's website has been developed using the J2EE framework. J2EE is a set of the specification which defines the standard for developing multi-tier enterprise applications with Java. The J2EE platform provides a complete framework for design, development, assembly, and deployment of Java applications built on multi-tiered distributed application model [12]. 
Groupon also used Nginx, a web server that provides load balancer that can improve load capacity of the website [13]. Since Groupon is a website that received a lot of visitors and customers, therefore Nginx must be deployed as to ensure the stability of the servers. This will prevent the server become overload from the intense visit of users.

Groupon does not provide searching functionality. Therefore customers need to browse through listed deals rather than search for a specific offer. Searching functionality is a must as it will make sure customers can find what they want or desire. By making this functionality absent from the mobile app, Groupon only allows the "Shopping" and "Travel" categories to be filtered by subdivisions. Nevertheless, Groupon managed to display adverts by limiting the quantity for each post. By using this concept, each post can only be load if user scrolls to bottom of the page. This process will reduce the load time and the use of data bandwidth. Groupon also has a deal notification, which allows customers to be notified of current promotions on their smartphone. The notification will appear on the top bar that level with the battery icon. This functionality is a big deal for customers since they need to keep updated with the latest promotions since Groupon need to make sure their customers will keep using Groupon to purchase things online. Moreover, Groupon also provides a countdown timer functionality that shows an amount of time limits for each advert. This time limit will be set by the vendors. The purpose of this functionality is it will make customers keep alert and at the same time, they can plans ahead of their schedule for shopping. This function also will urge the customers to take action.

LivingSocial is an electronic commerce marketing firm that offers daily deals to a wide customer base through emails and Internet advertising [14]. To get the promotion on the website, the firm will contact the business owner and propose them to join and promote their products with the reasonable discount rate. LivingSocial also will make a deal with business owner as to decide what kind of promotion that will be offered to the customer. If they manage to reach an agreement, the promotions will be up on the website. This promotion will be promoted inside the local city where the business owner located. For those who subscribe into that local city' newsfeed, they will receive the promotion through email. This promotion has their own limited time validation and varies for each product. Customers can get a voucher that can be redeemed at the store after they purchase the product from LivingSocial. By redeeming this voucher, LivingSocial can get their commission from the redeemed voucher, and the rest of the money will be given to store owner. LivingSocial's website has been developed using Ruby on Rails web framework. Ruby on Rails is a framework written in Ruby which developers could use Ruby to create web applications. Rails is based on the Model - View - Controller (MVC) design pattern for application architecture. MVC is a simple yet powerful pattern that has been implemented in many frameworks including the recent Microsoft ASP.NET MVC. The MVC pattern is easy to comprehend, and its implementation in Ruby on Rails is easy to follow. MVC also has the advantages of using their own libraries, where programmers do not need to code some functionality. LivingSocial uses geo-targeting that will provide their customers with deals from retailers in their local cities by using the IP address of their customer's computer or smartphone to locate where the signal is being sent from and thus where the person currently resides. This will allow customers to see total deals available in their area which is a great opportunity for retailers to promote their business. Plus, deals that receive a good feedback, high purchased, good recommendation and recommended by the author will be top on the list. Furthermore, customers would love to receive promotions or discount that based on their location [15].

LivingSocial also lists all the promotions that available, but this is quite tedious if there are lots of advertisements been advertised and customers need to scroll all the way down. This will lead the user to abandon the website. This problem can be solved if LivingSocial uses the pagination process of dividing content into discrete pages. Plus, LivingSocial can use a load button that can list out the product if the user wants it. Therefore, this will give a neat and user-friendly view inside LivingSocial's application. LivingSocial can limits the quantity of each advert displayed, and whenever customers scroll down, there will be loading function that load more adverts. The interface for the details information is quite clumsy. For example, if customers click on the advertisement, they will be directed to another page. This will make the page load longer than usual. LivingSocial makes the menu button disappear if customers scroll down. However, the menu button completely disappears, even after user scrolls it back to the top. This problem can be solved if the LivingSocial make the menu bar position is fixed and not hidden to its place, even customers scroll the page.

Tagme is an application that can help the customer to share the shopping experience on the social network with their friend. There are two mobile applications that have been compared and reviewed in this section. Thus, Tagme was selected since the application provides special key features such as allow customers to tag their friends during shopping. Plus, with the concept of gamification [16], Tagme will allow users to get rank or make to the top of the leaderboard based on their activities such as tagging, login, redeem vouchers and rewards. This type of gamification will allow the increment of engagement inside Tagme. This concept was applied by Waze, a popular GPS application that is rewarding their users after they shared information such as accidents, traffic jam or roadblock. Waze users will be rewarded with a collection of badges and opportunity to climb into higher level [17]. Therefore, by using this method, this will increase the user activities and engagement inside Waze. This technique is applied inside Tagme after the customers tagged their friends, users can collect points and redeem it later on to get a reward. The web-based of Tagme will allow the retailer to view analytic reports, traffic analysis and update latest promotions. By getting this analytical data, retailers can have a better insight regarding their business's performance. This all shall be done via webbased due to efficiency working performances. Plus, it will allow the retailers to create their own store and manage all their promotion in one place. 


\section{Implementation Method}

There are two categories for user requirements which are the businesses and the customer. From the businesses side, the modules that will be implemented including Register, Add Store, Add Advertisement and Manage Advertisement. This businesses side is developed for the retailer. The retailer can have full access control of their business from system dashboard. Inside Add Store module, the retailer will be enabled to insert store details such as store's name, operating time, location and category. After the store created by the retailer, they can add a new advertisement under selected store. This function is referring to Add Advertisement module. The creation of advertisement consists of title, price, and date. For Manage Advertisement module, the retailer can edit and delete their advertisements at will.

From the customer side, the modules are Register, Manage Advertisement and Tag Friends. Inside Manage Ads module, the customer can view and comment inside an advertisement that has been listed. Tag Friends module allow the customer to tag their friend with the current advertisement. By tagging their friend, the customer will receive points and can be redeemed to get a reward.

This section focuses on clarifying the implementation of the web application and hybrid application. Some software had already installed which is Bracket, Android Studio, Xampp, and FileZilla. This software is a basic tool for developing the website and the application. Android Studio is a platform for developing an Android application and currently replacing the Eclipse Android Development Tools as a major Android application development. By using Android Studio, it will serve as the container of the web view. This web view will be embedded with remote url. Inside this remote url, it contains the link to the website. For programming language inside Android Studio, Java and XML will be used. Xampp is a software that provides crossplatform web server solution that consists of web server application such as Apache HTTP Server, MariaDB database and scripting languages which is PHP and PERL. Xampp is installed as to build the localhost server inside a personal computer. Filezilla is a File Transfer Protocol (FTP) application that provides cross-platform functionality and supports FTP, SFTP, and FTPS (FTP over SSL/TLS). The main purpose of Filezilla is to upload and download files from web hosting server. This system is using PHP Hypertext Preprocessor (PHP) programming language, while MySQL language is used to connect the system to the database. Essentially, the suitable system interfaces are used for making easy of the interaction of the system.

The bracket is used as a platform for doing a PHP programming language to develop a user interface for the web-based system, connecting the database and the other system functions. Meanwhile, Bootstrap 3.0 is used as the template for the web system and hybrid app. Bootstrap is a suitable framework for developing this project because of its nature as responsive web design. Responsive web design is a concept that allowing the web pages of the desktop to be viewed like an application's interface. This will make the user interface becomes more user-friendly.

Fig. 1 shows the promotions in the form of vouchers and discount offers by the retailers around Batu Pahat, Johor. To get the offers, the user needs to tag their friends first. The process is repeated when the tagged friends want to get the offer by using this model; the offers can go viral to many intended customers.

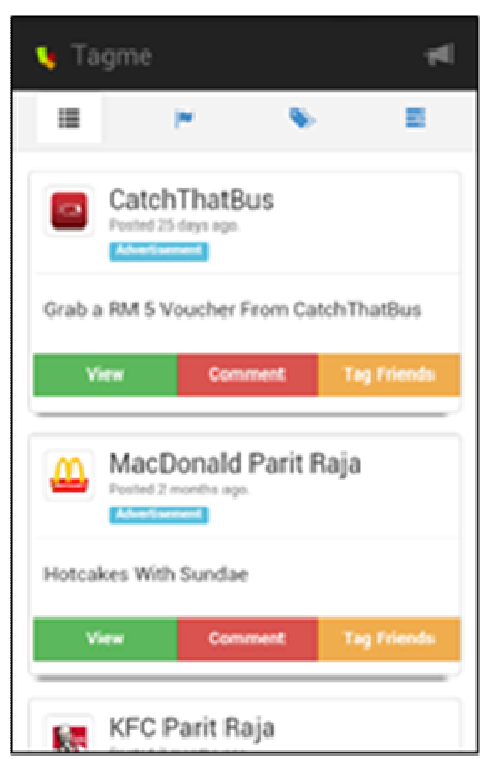

Fig. 1 Promotions display from retailers

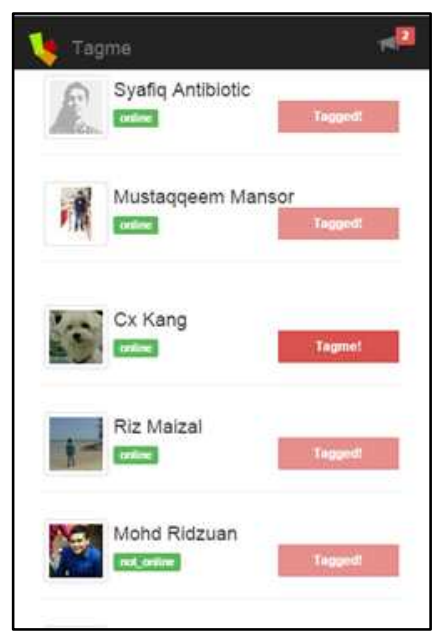

Fig. 2 Tagging friends page

Fig. 2 shows the page on tagging the friends on offer. The list of users comes from Facebook's friend list and the users that install the apps. By having all this information on the smartphone, users can plan ahead what to buy and where to eat during the shopping trip. This will save their time and money. All friends that have been tagged will be informed via email or notification icon. This notification icon will appear at the top right corner of the screen. Once the notification is clicked, they will be redirected to the voucher. Upon tagging friends with vouchers, users will receive points that will be accumulated as a reward that can be redeemed later on. This feature is a must since it will encourage the user to tag more voucher to their friends in order to receive points and rewards. The tagged friends will be marked as tagged. They can tag their friend again with other new promotion. This will encourage user engagement inside Tagme. 


\section{The System Coding (Hybrid Application)}

For a hybrid application, Android Studio will be used to serve as a container for the web content of Tagme. Hybrid mobile applications are built in a similar manner as websites. Both use a combination of technologies like HTML, CSS, and JavaScript. For front-end development, the Bootstrap framework will be used since it offers responsive web functionality. Web responsive is a functionality that allows the website to display the content according to the platform such as web browser or mobile browser. However, instead of targeting a mobile browser, hybrid applications target a web view that hosted inside a native container. This enables them to do things like access hardware capabilities of the mobile device such as camera, geo-location, and gallery. But still, the hybrid application still cannot access some major native hardware capabilities such as pop-up notification and Bluetooth functionality due to the limited resources and hardware compatibilities. This container was built using Java programming. By using Android Studio, all the web content can be load from remote url. This url is a link to the website that is embedded inside the Java programming. All the data will be synchronized since the website is directly displayed inside the container. Therefore, by using this method, the app will display the content of the website. Inside the screen of the app, there will be an android activity. Therefore, the activity of the whole main application will be contained the web view.

\section{RESULTS AND DISCUSSION}

The evaluation was carried out by testing all the functionality of the system to ensure that all system functions run smoothly. In addition, system testing was also carried out to identify the error arising. The process of testing is focused on three plans namely the test cases, the expected result and actual results.

\section{A. Test Cases}

Below are the test cases that have been performed on Tagme:

- User clicks "Tag Friend" to tag their friend.

- User can grab point when tagging their friends with the latest advert.

- User can comment to each advert.

- User can view others comment.

- User can view voucher and grab it.

- User can redeem voucher by using code.

All test cases were successfully performed on the apps.

\section{B. Testing User Acceptance}

The user acceptance testing is done by distributing questionnaire. The questionnaire distributed to 100 users. The form has various test cases and questions, including functions for each case.

Fig. 3 shows the results from the questionnaires whether Tagme provides enough information regarding events and advertisement that happens to users. $72 \%$ of the respondent has agreed that whether Tagme provides enough information regarding events and advertisement. This is because all the respondents can view each events detail that has been provided inside Tagme application.

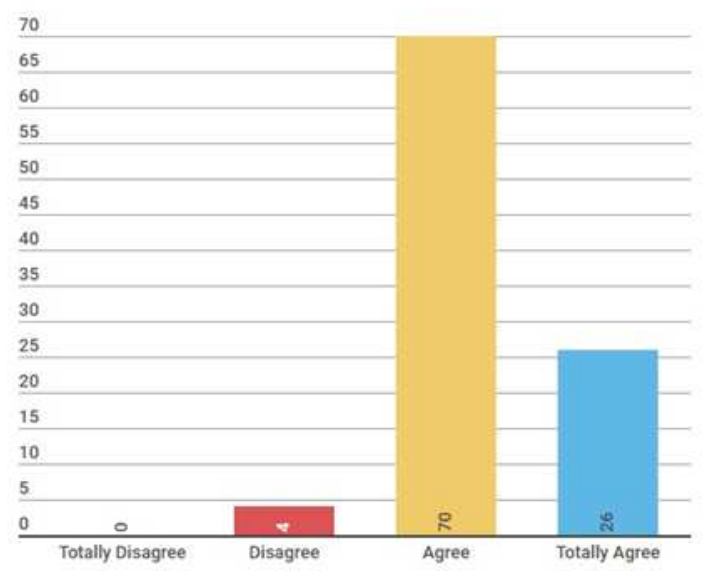

Fig. 3 User acceptance on Tagme covers all the required information about events happening around them

The bar chart in Fig. 4 shows the result of whether Tagme has an interesting design and easy to use interface. $70 \%$ respondents agree while $26 \%$ respondents also strongly agree with the statement. However, $4 \%$ respondents disagree with the statement. An interesting and easy to use interface is important to keep users using the apps.

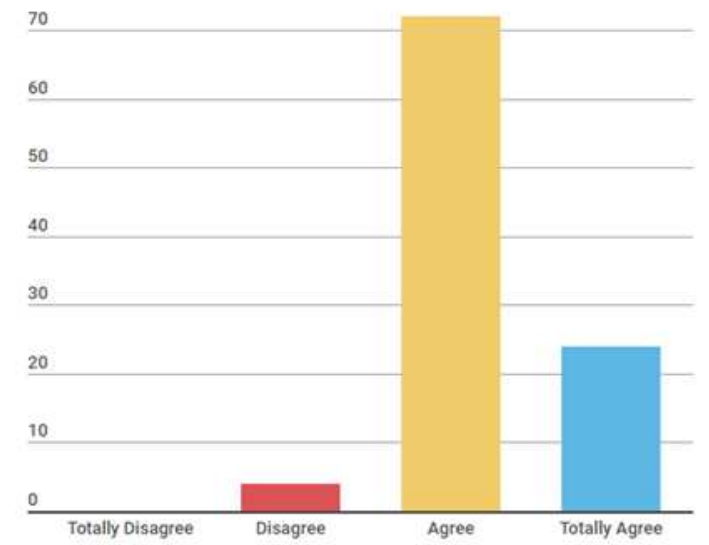

Fig. 4 User acceptance on Tagme design and interface

\section{CONCLUSIONS}

Current vouchers giveaways system did not encourage their users to promote current offers to others. By using Tagme, it will allow the user to tag their friends first on the offer. With the use of global positioning system (GPS) inside every smartphone, we will add a new way to notify every user with current promotion based on their location. The advantage of this method is user can enjoy every deals and promotion around them without a need to explore the store by themselves. Therefore, the user can save time and energy. Plus, retailers can save their money from spending on flyers or brochure to promote their product since all of this can be done via a pop-up notification. This is a crucial feature since it will target customers within promotion's range.

We proposed another method to attract customers into offers by using the social networking. Furthermore, they will receive points based on total tagged friends, and this points can be redeemed later on to get any reward that available. Plus, the offer can go viral on their inside and 
outside the circle of friends. For future works, we will add how to suggest which friends to tag based on their usage history. This is to ensure that the offer will go to the user that really interested in them in order to increase sales for businesses.

\section{ACKNOWLEDGMENT}

This work is sponsored by Universiti Tun Hussein Onn Malaysia under MDR Grant U095 and also supported by GATES IT Solution Sdn. Bhd. under its publication scheme.

\section{REFERENCES}

[1] Mahdin, H., Omar, A.H., Yaacob, S.S., Kasim, S. and Fudzee, M.F.M., 2016, November. Minimizing Heatstroke Incidents for Young Children Left inside Vehicle. In IOP Conference Series: Materials Science and Engineering (Vol. 160, No. 1, p. 012094). IOP Publishing.

[2] Kasim, S., Azahar, U.A., Samsudin, N.A., Fudzee, M.F.M., Mahdin, H., Ramli, A.A. and Suparjoh, S., 2016, August. E-Code Checker Application. In International Conference on Soft Computing and Data Mining (pp. 570-578). Springer, Cham.

[3] Ishak, I., Sidi, F., Jabar, M.A., Sani, N.F.M., Mustapha, A. and Supian, S.R., 2012. A survey on security awareness among social networking users in malaysia. Australian Journal of Basic and Applied Sciences, 6(12), pp.23-29.

[4] Ahmad, M., Hussin, N., Zulkarnain, S., Mahdin, H. and Fudzee, M.F.M., 2016, August. Factors Influencing the Use of Social Media in Adult Learning Experience. In International Conference on Soft Computing and Data Mining (pp. 579-588). Springer, Cham.

[5] Lin, J., Oentaryo, R.J., Lim, E.P., Vu, C., Vu, A., Kwee, A.T. and Prasetyo, P.K., 2016, March. A business zone recommender system based on Facebook and urban planning data. In European Conference on Information Retrieval (pp. 641-647). Springer International Publishing.

[6] Edelman, B., Jaffe, S. and Kominers, S.D., 2016. To groupon or not to groupon: The profitability of deep discounts. Marketing Letters, 27(1), pp.39-53.
[7] Vijayalakshmi, S., \& Mahalakshmi, V. (2013). An impact of consumer buying behavior in decision making process in purchase of electronic home appliances in Chennai (India): an empirical study. Elixir Marketing Management, 59, 15267-15273.

[8] Hutter, K., Hautz, J., Dennhardt, S., \& Füller, J. (2013). The impact of user interactions in social media on brand awareness and purchase intention: the case of MINI on Facebook. Journal of Product \& Brand Management, 22(5/6), 342-351.

[9] Swamynathan, G., Wilson, C., Boe, B., Almeroth, K. and Zhao, B.Y., 2008, August. Do social networks improve e-commerce?: a study on social marketplaces. In Proceedings of the first workshop on Online social networks (pp. 1-6). ACM.

[10] Jain, N., 2015. Review of different responsive CSS Front-End Frameworks. Journal of Global Research in Computer Science, 5(11), pp.5-10.

[11] Liu, Y. and Sutanto, J., 2012. Buyers' purchasing time and herd behavior on deal-of-the-day group-buying websites. Electronic Markets, 22(2), pp.83-93.

[12] Nagappan, R., Skoczylas, R. and Sriganesh, R.P., 2003. Developing Java web services: architecting and developing secure web services using Java. John Wiley \& Sons.

[13] Nedelcu, C. (2010). Nginx HTTP Server: Adopt Nginx for Your Web Applications to Make the Most of Your Infrastructure and Serve Pages Faster Than Ever. Packt Publishing Ltd.

[14] Hughes, S. and Beukes, C., 2012. Growth and implications of social e-commerce and group buying daily deal sites: The case of Groupon and LivingSocial. The International Business \& Economics Research Journal (Online), 11(8), p.921.

[15] Bhave, K., Jain, V., \& Roy, S. (2013). Understanding the orientation of gen $\mathrm{Y}$ toward mobile applications and in-app advertising in India. International Journal of Mobile Marketing, 8(1).

[16] O'Donovan, S., Gain, J., \& Marais, P. (2013, October). A case study in the gamification of a university-level games development course. In Proceedings of the South African Institute for Computer Scientists and Information Technologists Conference (pp. 242-251). ACM.

[17] Lambruschini, B. B., \& Pizarro, W. G. (2015, July). TechGamification in university engineering education: Captivating students, generating knowledge. In Computer Science \& Education (ICCSE), 2015 10th International Conference on (pp. 295-299). IEEE. 